\title{
The incision strategy for minimizing sural nerve injury in medial displacement calcaneal osteotomy: a cadaveric study
}

\author{
Jeong-Hyun Park ${ }^{1 \dagger}$, Kwang-Rak Park ${ }^{1 \dagger}$, Digud Kim, Hyung-Wook Kwon' ${ }^{1}$, Mijeong Lee ${ }^{1}$, Yu-Jin Choi ${ }^{1}$, \\ Yong-Been $\mathrm{Kim}^{2}$, Suyeon Park ${ }^{3}$, Jinseo Yang $^{4}$ and Jaeho $\mathrm{Cho}^{2^{*}}$ (D)
}

\begin{abstract}
Background: The skin incision for medial displacement calcaneal osteotomy (MDCO) often damages the sural nerve. We aimed to identify the practical reference area in which the surgeon can incise the skin to minimize the injury of the sural nerve during MDCO.

Methods: The foot and ankles of 20 cadavers were dissected. The landmarks were the following four anatomical references: point $A$, the tip of the lateral malleolus; point $B$, the inferior margin of the calcaneus on the vertical line through point $A$; point $C$, the posteroinferior apex of the calcaneus; and point $D$, the lateral border of the Achilles tendon on the horizontal line through point $A$. The distances from the sural nerve to points $A$ and $B$ in the vertical direction (lines D1 and D2, respectively), to points $A$ and $C$ in the diagonal direction (lines D3 and D4, respectively), and to points $A$ and $D$ in the horizontal direction (lines D5 and D6, respectively) were measured.
\end{abstract}

Results: The median ratios of D1 to D1+D2, D3 to D3+D4, and D5 to D5+D6 were 0.34 (range 0.25 to 0.45 ), 0.23 (range 0.16 to 0.33 ), and 0.38 (range 0.26 to 0.50 ), respectively.

Conclusions: The distance ratios according to easily identifiable references would be a more practical incision strategy for surgeons to minimize sural nerve injury in both open and minimally invasive/percutaneous MDCO.

Keywords: Cadaver study, Calcaneus, Osteotomy, Incision, Sural nerve

\section{Introduction}

Medial displacement calcaneal osteotomy (MDCO) corrects hindfoot valgus by increasing the medial pull of the Achilles tendon by moving the posterior part of the calcaneus medially [15]. Historically, this procedure was the most common method to treat adult acquired flatfoot deformity (AAFD) with stage II tibialis posterior tendon insufficiency, in combination with other surgical procedures [8]. The osteotomy is usually performed in a direction perpendicular to the calcaneus axis or $45^{\circ}$ to the plantar aspect of the hindfoot. Clinical and cadaveric studies have reported that achievement of at least 10

\footnotetext{
* Correspondence: hohotoy@nate.com

† Jeong-Hyun Park and Kwang-Rak Park contributed equally to this work.

2Department of Orthopaedic Surgery, Chuncheon Sacred Heart Hospital, Hallym University, 77, Sakju-ro, Chuncheon-si, Gangwon-do 200-704, Republic of Korea

Full list of author information is available at the end of the article
}

$\mathrm{mm}$ of displacement can change ankle contact pressures and joint distribution $[5,8]$.

To achieve an adequate displacement of the posterior calcaneus, an open procedure is traditionally performed through the skin incision in line with the osteotomy [11, 17]. Common complication of this procedure includes delayed wound healing, wound infection, and sural nerve damages $[1,3,16,20]$. The sural nerve is vulnerable to injury during the initial incision because it runs superficially along the lateral aspect of the calcaneus. Evidence of iatrogenic sural nerve-related symptoms following open approach for MDCO have been reported at 6.8$25 \%[1,3,4,16,20]$. The minimally invasive calcaneal osteotomy (MICO) technique has recently emerged to reduce the incidence of wound complications; nevertheless, the sural nerve may be exposed to greater risk of iatrogenic injury because of limited visualization.

(c) The Author(s). 2019 Open Access This article is distributed under the terms of the Creative Commons Attribution 4.0 International License (http://creativecommons.org/licenses/by/4.0/), which permits unrestricted use, distribution, and 
A cadaveric study by Talusan et al. [19] defined extension lines from the calcaneal tuberosity to the plantar fascia origin as landmarks for the identifying safe zone to minimize sural nerve damage in MDCO. Other authors described the position of the skin incision using the sural nerve relationship to the Achilles tendon, with the lateral malleolus as a reference point [2, 18, 21]. However, it is somewhat insufficient to determine the optimal incision area to minimize damage to the sural nerve with only one landmark or two reference points in both open and minimally invasive medial displacement osteotomies. Only one recent cadaveric study has described the course of the sural nerve as the relationship of four anatomical reference points [7].

Therefore, we aimed to describe the anatomical course of the sural nerve in relation to the easily identifiable landmarks at the time surgery and verify the relationship between sural nerve and reference points. We hypothesized that a practical reference area could be provided to minimize injury of the sural nerve during MDCO, regardless of the incision methods.

\section{Materials and methods}

The cadavers used in the present study were donated to our institutions with consent for education and research. In addition, this study was approved by the Ethics Committee of Chuncheon Sacred Heart Hospital, Hallym University (Institutional Review Board number: 201795).

Twenty foot and ankle specimens in adult formalinfixed cadavers were dissected. Of the 20 specimens, 10 (50\%) were from females and 10 (50\%) were from males. The mean age of the donors at death was 73.9 (range, 49-91) years. The lateral aspect of the foot of all cadavers had intact skin with no signs of previous trauma or surgery, obvious deformities, and/or ulcers.

The foot and ankle specimens were stabilized in the lateral position, and the following four reference points described by Geng et al. [7] were identified by palpation and marked before dissection: point $\mathrm{A}$, the tip of the lateral malleolus; point $B$, the inferior margin of the calcaneus on the vertical line through point $A$; point $C$, the posteroinferior apex of the calcaneus; and point $\mathrm{D}$, the lateral border of the Achilles tendon on the horizontal line through point A (Fig. 1).

A section of the skin was meticulously dissected from the lateral side of the ankle and hindfoot. The posterior inferior margin of the skin dissection was started at the most inferior and posterior points of the calcaneus. Then, the skin and subcutaneous tissue of the lateral part of the ankle and hindfoot were carefully dissected. This approach provided adequate visibility to confirm the anatomical structure around the sural nerve, including the main trunk of the sural nerve, the lateral

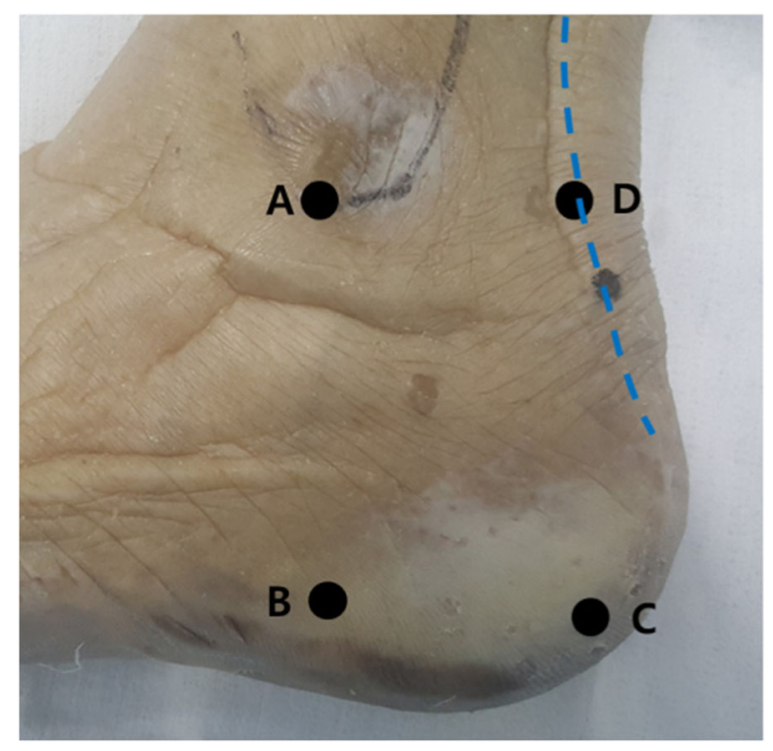

Fig. 1 Four reference points on the skin marked before dissection. Point $A$, the tip of the lateral malleolus; point $B$, the inferior margin of the calcaneus on the vertical line through point $A$; point $C$, the posteroinferior apex of the calcaneus; and point $\mathrm{D}$, the lateral border of the Achilles tendon (blue dotted line) on the horizontal line through point A

calcaneal branches, and the small saphenous vein running along with the sural nerve.

After a careful dissection, the distances from the sural nerve to points $A$ and $B$ in the vertical direction (lines $\mathrm{D} 1$ and D2, respectively), to points $\mathrm{A}$ and $\mathrm{C}$ in the diagonal direction (lines D3 and D4, respectively), and to points A and D in the horizontal direction (lines D5 and D6, respectively) were measured using a surgical ruler (Fig. 2). The identification of landmarks and measurements of the distances were performed independently by two researchers. Each independent researcher repeatedly measured the distance twice after identifying the landmark. The averages of the two researchers' measurements were adopted as the values to describe each specimen.

\section{Statistical analysis}

Inter- and intraobserver reliabilities for all measurements were calculated using the intraclass correlation coefficient (ICC). To evaluate the central tendency of all specimens, each distance was presented using the median and range. The ratios were calculated, and each ratio was presented as the median, range, and interquartile range. A Spearman correlation analysis was performed to determine the relationship between the measurements. We used scatter plots to visually represent the relationships. The Wilcoxon-Mann-Whitney test was performed to compare the ratio between males and females, while the Spearman correlation analysis was 


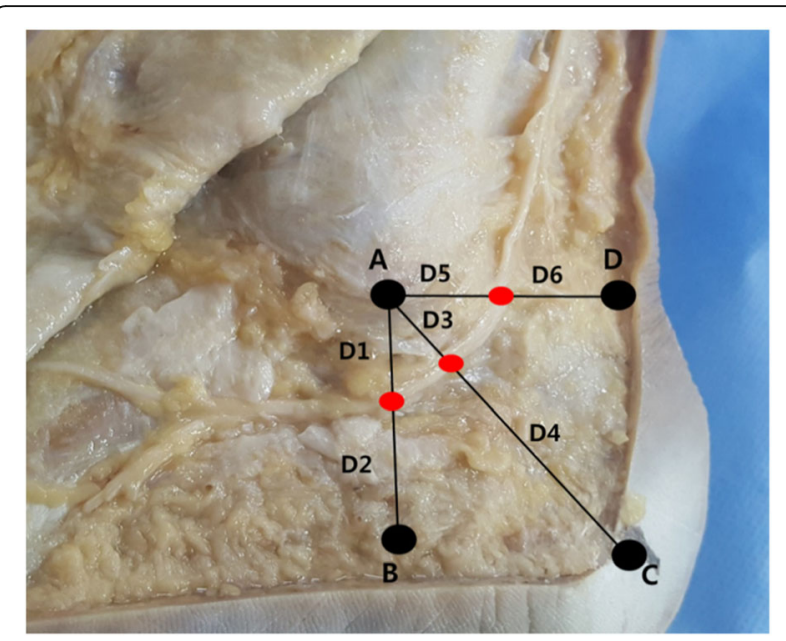

Fig. 2 The distances were measured. Line D1, the vertical distance from point $A$ to the sural nerve; line $D 2$, the vertical distance from the sural nerve to point $B$; line D3, the diagonal distance from point A to the sural nerve; line D4, the diagonal distance from the sural nerve to point $C_{\text {; }}$ line D5, the horizontal distance from point A to the sural nerve; and line D6, the horizontal distance from the sural nerve to point $D$

performed to evaluate the relationship between ratio and age. All statistical analyses were performed using SPSS 25.0 software (SPSS, Chicago, IL), and $P$ values less than .05 were considered statistically significant.

\section{Results}

Intraclass correlation coefficients were generated for all measurements. All measurements were greater than 0.8 (indicating acceptable reliability) and were employed in the study. The median values, ranges, and interquartile ranges (IQR) for all distances measured between the sural nerve and the selected reference landmarks are listed in Table 1. Mean values and standard deviations were also provided to help compare with the distances

Table 1 The distances measured between the sural nerve and the selected reference landmarks ( $n=20$ specimens)

\begin{tabular}{|c|c|c|c|c|c|c|c|}
\hline \multirow{2}{*}{$\begin{array}{l}\text { Distance } \\
(\mathrm{mm})\end{array}$} & \multirow[t]{2}{*}{ Median } & \multicolumn{2}{|c|}{ Range } & \multicolumn{2}{|l|}{ IQR } & \multirow[t]{2}{*}{ Mean } & \multirow[t]{2}{*}{ SD } \\
\hline & & Min & Max & Q1 & Q3 & & \\
\hline D1 & 13.00 & 10.00 & 21.00 & 11.25 & 18.00 & 14.45 & 3.78 \\
\hline D2 & 27.50 & 21.00 & 32.00 & 24.00 & 30.75 & 27.20 & \\
\hline D3 & 13.00 & 9.00 & 18.00 & 10.50 & 14.00 & 12.90 & \\
\hline 44 & 41.50 & 27.00 & 53.00 & 35.50 & 46.50 & 41.45 & \\
\hline D5 & 15.00 & 8.00 & 24.00 & 12.25 & 19.75 & 15.65 & \\
\hline D6 & 27.50 & 13.00 & 39.00 & 20.50 & 30.00 & 25.90 & \\
\hline
\end{tabular}

$\mathrm{D} 1$, the vertical distance from point $A$ to the sural nerve; $D 2$, the vertical distance from the sural nerve to point $B ; D 3$, the diagonal distance from point $A$ to the sural nerve; $D 4$, the diagonal distance from the sural nerve to point $C$; $\mathrm{D} 5$, the horizontal distance from point $A$ to the sural nerve; D6, the horizontal distance from the sural nerve to point $\mathrm{D} ; \mathrm{IQR}$, interquartile ranges; $\mathrm{Q} 1$, lower quartile; Q3, upper quartile; SD, standard deviation reported in previous studies. The median ratios of D1 to $\mathrm{D} 1+\mathrm{D} 2, \mathrm{D} 3$ to $\mathrm{D} 3+\mathrm{D} 4$, and $\mathrm{D} 5$ to $\mathrm{D} 5+\mathrm{D} 6$ were 0.34 (range 0.25 to 0.45 ), 0.23 (range 0.16 to 0.33 ), and 0.38 (range 0.26 to 0.50 ), respectively (Table 2 ). The median values, ranges, and IQR for all ratios are also shown in a box plot (Fig. 3).

The distance values measured from the lateral malleolar tip to the sural nerve in each direction of the reference points showed a statistically significant correlation with each value and a relatively high correlation coefficient (Fig. 4). All ratios (R1, R3, and R5) showed statistically significant correlations with each ratio and relatively high correlation coefficients (Fig. 5).

There was no significant difference in all ratios by gen$\operatorname{der}(p=0.579$ [R1], 0.280 [R3], and 0.165 [R5]). The correlation between age and all ratios was not significant $(p=0.462$ [R1], 0.221 [R3], and 0.163 [R5]).

\section{Discussion}

Medial displacement calcaneal osteotomy (MDCO) is commonly used concurrently with other osteotomies and tendon transfers in the treatment of adult acquired flatfoot deformity (AAFD). Traditionally, the procedure includes an oblique incision on the lateral heel. A common complication is sural nerve damage around the incision $[1,16,20]$. Although minimally invasive or percutaneous techniques have recently emerged in the hope of minimizing incisional complications, the risk of iatrogenic damage to the sural nerve remains $[6,9,19]$.

Because the sural nerve runs superficially on the lateral aspect of the heel [14], it is theoretically vulnerable to injury during the incision if an inappropriate incision is made. In order to minimize damage to the sural nerve in both open and minimally invasive/percutaneous medial displacement osteotomies, it is of utmost importance that an adequate skin incision is made. In our study, the reference points of the anatomical structure that can be easily recognized on the skin surface during surgery were described in relation to the anatomical course of the sural nerve.

Previous studies have also detailed the sural nerve in relation to anatomical landmarks. Solomon et al. [18] used two points corresponding to the tip and the most prominent posterior part of the lateral malleolus as

Table 2 The ratios calculated based on the measured distance values ( $n=20$ specimens)

\begin{tabular}{lllllll}
\hline Ratio & Median & \multicolumn{2}{l}{ Range } & & \multicolumn{2}{l}{ IQR } \\
& & Min & Max & & Q1 & Q3 \\
\hline R1 & 0.34 & 0.25 & 0.45 & & 0.29 & 0.40 \\
R3 & 0.23 & 0.16 & 0.33 & & 0.21 & 0.27 \\
R5 & 0.38 & 0.26 & 0.50 & & 0.33 & 0.42 \\
\hline
\end{tabular}

$\mathrm{R} 1$, ratio of $\mathrm{D} 1$ to $\mathrm{D} 1+\mathrm{D} 2$; $\mathrm{R} 3$, ratio of $\mathrm{D} 3$ to $\mathrm{D} 3+\mathrm{D} 4$; $\mathrm{R} 5$, ratio of $\mathrm{D} 5$ to $\mathrm{D} 5+\mathrm{D} 6$; $\mathrm{IQR}$, interquartile ranges; $\mathrm{Q} 1$, lower quartile; $\mathrm{Q} 3$, upper quartile 




Fig. 3 Box plot of the all ratios ( $n=20$ specimens). The boxes represent the interquartile ranges; the thick horizontal line inside the boxes represents the median values; the horizontal lines above and below the boxes represent the ranges. R1, ratio of D1 to D1+D2; R3, ratio of $\mathrm{D} 3$ to $\mathrm{D} 3+\mathrm{D} 4$; $\mathrm{R} 5$, ratio of $\mathrm{D} 5$ to $\mathrm{D} 5+\mathrm{D} 6$

reference points. Kammar et al. [10] presented four arbitrary points as references at a certain distance from the point of insertion of the Achilles tendon into the calcaneus. Talusan et al. [19] defined extension lines from the calcaneal tuberosity to the plantar fascia origin as the baseline. We believe that these reference points (only two points, arbitrary points on the Achilles tendon and a single baseline) are insufficient to provide detailed reference information to determine the optimal incision site to avoid damage to the sural nerve during MDCO. We believe the four landmarks applied in this study would be more useful in describing the course of the sural nerve.

We used a total of four reference points (the tip of the lateral malleolus, the inferior margin of the calcaneus, the posteroinferior apex of the calcaneus, and the lateral border of the Achilles tendon) that can be easily recognized on the skin surface during surgery. In previous studies $[2,7,18]$, the mean vertical distances between the tip of the lateral malleolus and the sural nerve were reported as $13.15 \pm 6.88,13 \pm 7$, and $13.1 \pm 4.1 \mathrm{~mm}$, respectively, compared with $13 \pm 3.78 \mathrm{~mm}$ in our study. These differences in values were not significant and may be accounted for by the size and race of the cadavers and the variability of the nerve course. We therefore determined that it would be more reliable and clinically useful to describe the ratio rather than the absolute distance. Table 2 and Fig. 3 show that the maxima of the ratios (R1, R3, and $\mathrm{R} 5$ ) approximate to $0.45,0.33$, and 0.50 , respectively. Using these maximum ratio values through reference landmarks based on the tip of the lateral malleolus prior to surgical incision, we believe that an incision area for sural nerve preservation can be established during MDCO (Fig. 6).

Geng et al. [7] reported the maximum of the ratios applied in the same manner as this study, with slightly different values except for R3. The authors suggested that skin incision for MDCO can be determined using a reference point that is one third (or not exactly $32 \%$ ) of the distance from the tip of the lateral malleolus to the posteroinferior margin of the calcaneus; however, the use of vertical and horizontal lines between reference points was not mentioned. Our results showed that both distance and ratio values measured from the lateral malleolar tip to the sural nerve in each direction of the reference points had significant correlations with each value. Therefore, we believe that this study, presenting up to auxiliary reference points in the vertical and horizontal directions, would more accurately account for the incision criteria that minimize the damage of the sural nerve during MDCO.



(B)

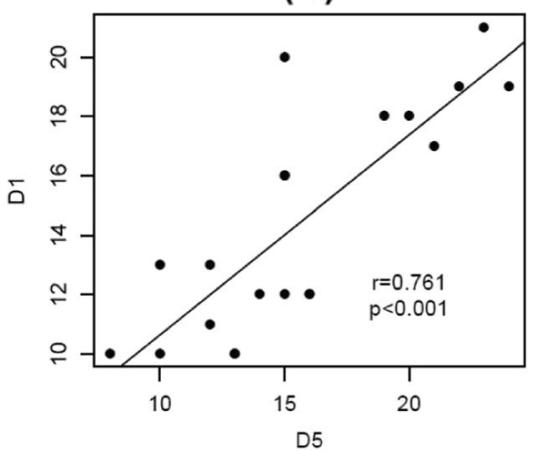

(C)

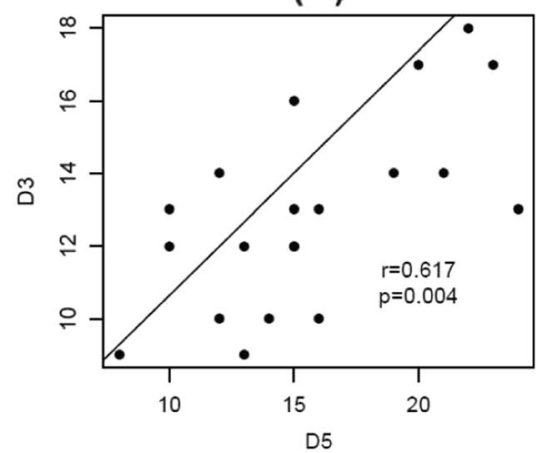

Fig. 4 Scatter plot to visually represent the relationships between the distances. D1, the vertical distance from the lateral malleolar tip to the sural nerve; D3, the diagonal distance from the lateral malleolar tip to the sural nerve; D5, the horizontal distance from the lateral malleolar tip to the sural nerve 

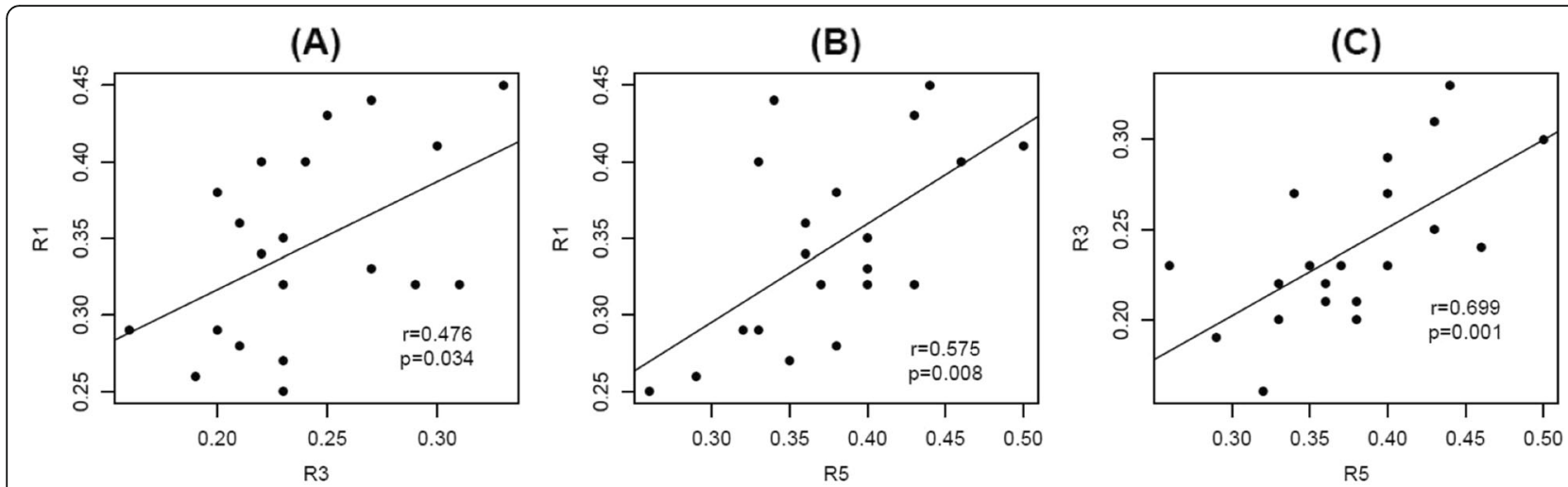

Fig. 5 Scatter plot to visually represent the relationships between the ratios. R1, ratio of D1 to D1+D2; R3, ratio of D3 to D3+D4; R5, ratio of D5 to $\mathrm{D} 5+\mathrm{D} 6$

In open procedures for $\mathrm{MDCO}$, the oblique incision may be made equal to the osteotomy line at the back of more than one third of the line connecting the posteroinferior apex of the calcaneus from the tip of the lateral malleolus within the proposed incision criteria. Lee et al. [12] discussed the importance of selecting the entry point for the burr in minimally invasive procedures for MDCO; however, they did not propose an accurate entry point. Therefore, the proposed incision criteria in this study can be useful for minimizing iatrogenic damage to the sural nerve in both open and minimally invasive/percutaneous medial displacement osteotomies. Furthermore, there was no significant difference in the results of this study by gender. Madhavi et al. [13] reported that

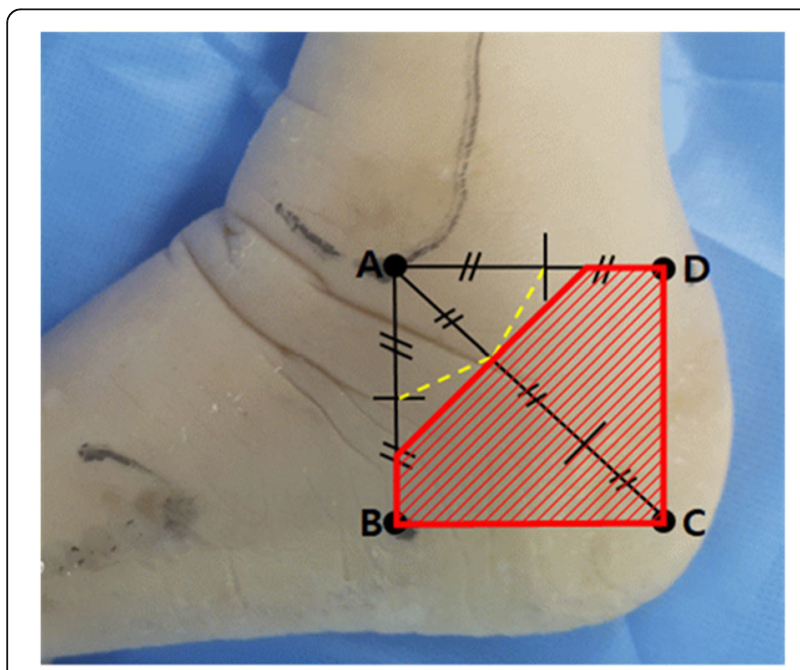

Fig. 6 The incision area for minimizing sural nerve injury in MDCO. The yellow dotted line represents the baseline of distance from the lateral malleolar tip to the sural nerve. The red hatched area represents a practical zone for clinical applications, including more than one half of the horizontal distance between points $A$ and $B$, more than one half of the vertical distance between point $A$ and $D$, and more than one third of the diagonal distance between point $A$ and $C$ there was no association between sural nerve distribution type and gender in the foot. Therefore, it would be possible to apply the proposed incision criteria in this study regardless of gender.

This study has some limitations. Differences in the incision area for minimizing sural nerve injury between embalmed cadaver and fresh cadaver or live human may exist due to stiffness of soft tissues and immobile joints in embalmed cadaver specimens. However, the cadaveric nature of this study did not allow us to detect such potential differences. Because the ankles of all embalmed cadaveric specimens were fixed at a neutral position of $90^{\circ}$, the measurement between the bony landmarks and the sural nerve was almost identical. The small sample size may have weakened the statistical power with respect to the possibility of sural nerve variations. For this reason, only the main trunk of the sural nerve was analyzed, and there may be an anatomical variation of the branches of the sural nerve. Larger samples are necessary for future studies to generate more accurate descriptions of the sural nerve. Furthermore, to clinically apply the results of this study, a validation study using a larger number of cadavers is required.

\section{Conclusions}

The distance ratios presented in this cadaveric study can be helpful to describe the course of the sural nerve according to easily identifiable bony landmarks during MDCO. We suggested skin incision criteria of more than one third of the distance from the tip of the lateral malleolus to the posteroinferior apex of the calcaneus as the main reference, and more than $1 / 2$ points from the tip of the lateral malleolus to the vertical and horizontal directions would be the auxiliary reference to minimize iatrogenic damage of the sural nerve during MDCO. Furthermore, the proposed incision areas are more practical reference points for minimizing sural nerve injury in both open and minimally invasive/percutaneous MDCO. 


\section{Supplementary information}

Supplementary information accompanies this paper at https://doi.org/10. 1186/s13018-019-1411-7.

Additional file 1: The datasets used and analyzed during the current study.

\section{Abbreviations}

AAFD: Adult acquired flatfoot deformity; ICC: Intraclass correlation coefficient IQR: Interquartile ranges; MDCO: Medial displacement calcaneal osteotomy; MICO: Minimally invasive calcaneal osteotomy

\section{Acknowledgements}

We thank Hongtae Kim and Dae Young Hur for contributing and collecting the cadavers.

\section{Authors' contributions}

JHP and JC contributed to the conception of the study. DK, HWK, ML, and YJC contributed to the investigation of the cadavers. YBK, SP, JY, and JC contributed to the formal analysis of the datasets. KRP and JC wrote the original manuscript. JHP, KRP, and JC reviewed and edited the manuscripts. All authors of the manuscript have read, approved, and agreed to its final content and are accountable for all aspects of the accuracy and integrity of the manuscript in accordance with ICMJE criteria.

\section{Funding}

This research was supported by Hallym University Research Fund 2019(HURF2019-23).

\section{Availability of data and materials}

The datasets used and analyzed during the current study are presented in Additional file 1.

\section{Ethics approval and consent to participate}

The cadavers used in the present study were donated to our institutions with consent for education research and publication. In addition, this study was approved by the Ethics Committee of Chuncheon Sacred Heart Hospital, Hallym University (Institutional Review Board number: 2017-95).

\section{Consent for publication}

The cadavers used in the present study were donated to our institutions with consent for education research and publication.

\section{Competing interests}

The authors declare that they have no competing interests. The funders had no role in the design of the study; in the collection, analyses, or interpretation of the data; in the writing of the manuscript; and in the decision to publish the results.

\section{Author details}

'Department of Anatomy \& Cell Biology, Graduate School of Medicine, Kangwon National University, Kangwon, Republic of Korea. ${ }^{2}$ Department of Orthopaedic Surgery, Chuncheon Sacred Heart Hospital, Hallym University, 77, Sakju-ro, Chuncheon-si, Gangwon-do 200-704, Republic of Korea. ${ }^{3}$ Department of biostatistics, College of Medicine, Soonchunhyang University Hospital, Seoul, Republic of Korea. ${ }^{4}$ Department of Neurosurgery, Chuncheon Sacred Heart Hospital, Hallym University, Chuncheon, Republic of Korea.

Received: 7 August 2019 Accepted: 14 October 2019

Published online: 12 November 2019

\section{References}

1. Abbasian A, Zaidi R, Guha A, Goldberg A, Cullen N, Singh D. Comparison of three different fixation methods of calcaneal osteotomies. Foot Ankle Int. 2013;34:420-5.

2. Aktan Ikiz ZA, Ucerler $\mathrm{H}$, Bilge $\mathrm{O}$. The anatomic features of the sural nerve with an emphasis on its clinical importance. Foot Ankle Int. 2005;26:560-7.

3. Catanzariti AR, Lee MS, Mendicino RW. Posterior calcaneal displacement osteotomy for adult acquired flatfoot. J Foot Ankle Surg. 2000;39:2-14.
4. Coetzee JC, Hansen ST. Surgical management of severe deformity resulting from posterior tibial tendon dysfunction. Foot Ankle Int. 2001:22:944-9.

5. Davitt JS, Beals TC, Bachus KN. The effects of medial and lateral displacement calcaneal osteotomies on ankle and subtalar joint pressure distribution. Foot Ankle Int. 2001;22:885-9.

6. Didomenico LA, Anain J, Wargo-Dorsey M. Assessment of medial and lateral neurovascular structures after percutaneous posterior calcaneal displacement osteotomy: a cadaver study. J Foot Ankle Surg. 2011;50:668-71.

7. Geng X, Xu J, Ma X, Wang X, Huang J, Zhang C, et al. Anatomy of the sural nerve with an emphasis on the incision for medial displacement calcaneal osteotomy. J Foot Ankle Surg. 2015;54:341-4.

8. Guha AR, Perera AM. Calcaneal osteotomy in the treatment of adult acquired flatfoot deformity. Foot Ankle Clin. 2012;17:247-58.

9. Jowett $C R$, Rodda D, Amin A, Bradshaw A, Bedi HS. Minimally invasive calcaneal osteotomy: a cadaveric and clinical evaluation. Foot Ankle Surg. 2016;22:244-7.

10. Kammar H, Carmont MR, Kots E, Laver L, Mann G, Nyska M, et al. Anatomy of the sural nerve and its relation to the Achilles tendon by ultrasound examination. Orthopedics. 2014;37:e298-301.

11. Kendal AR, Khalid A, Ball T, Rogers M, Cooke P, Sharp R. Complications of minimally invasive calcaneal osteotomy versus open osteotomy. Foot Ankle Int. 2015:36:685-90.

12. Lee M, Guyton GP, Zahoor T, Schon LC. Minimally invasive calcaneal displacement osteotomy site using a reference Kirschner wire: a technique tip. J Foot Ankle Surg. 2016;55:1121-6.

13. Madhavi C, Isaac B, Antoniswamy B, Holla SJ. Anatomical variations of the cutaneous innervation patterns of the sural nerve on the dorsum of the foot. Clin Anat. 2005;18:206-9.

14. Riedl O, Frey M. Anatomy of the sural nerve: cadaver study and literature review. Plast Reconstr Surg. 2013;131:802-10.

15. Saxena A, Patel R. Medial displacement calcaneal osteotomy: a comparison of screw versus locking plate fixation. J Foot Ankle Surg. 2016;55:1164-8.

16. Schuh R, Gruber F, Wanivenhaus A, Hartig N, Windhager R, Trnka HJ. Flexor digitorum longus transfer and medial displacement calcaneal osteotomy for the treatment of stage II posterior tibial tendon dysfunction: kinematic and functional results of fifty one feet. Int Orthop. 2013;37:1815-20.

17. Silva MG, Tan SH, Chong HC, Su HC, Singh IR. Results of operative correction of grade IIB tibialis posterior tendon dysfunction. Foot Ankle Int. 2015;36:165-71

18. Solomon LB, Ferris L, Tedman R, Henneberg M. Surgical anatomy of the sural and superficial fibular nerves with an emphasis on the approach to the lateral malleolus. J Anat. 2001;199:717-23.

19. Talusan PG, Cata E, Tan EW, Parks BG, Guyton GP. Safe zone for neural structures in medial displacement calcaneal osteotomy: a cadaveric and radiographic investigation. Foot Ankle Int. 2015;36:1493-8.

20. Wacker JT, Hennessy MS, Saxby TS. Calcaneal osteotomy and transfer of the tendon of flexor digitorum longus for stage-ll dysfunction of tibialis posterior. Three- to five-year results. J Bone Joint Surg Br. 2002;84:54-8.

21. Webb J, Moorjani N, Radford M. Anatomy of the sural nerve and its relation to the Achilles tendon. Foot Ankle Int. 2000:21:475-7.

\section{Publisher's Note}

Springer Nature remains neutral with regard to jurisdictional claims in published maps and institutional affiliations.

Ready to submit your research? Choose BMC and benefit from:

- fast, convenient online submission

- thorough peer review by experienced researchers in your field

- rapid publication on acceptance

- support for research data, including large and complex data types

- gold Open Access which fosters wider collaboration and increased citations

- maximum visibility for your research: over $100 \mathrm{M}$ website views per year

At BMC, research is always in progress.

Learn more biomedcentral.com/submissions 